MS007.P01

\title{
Synthesis and crystal structure of a new layered $\mathrm{CoBi}_{2} \mathrm{O}_{2} \mathrm{~F}_{4}$ oxofluoride
}

Eleni Mitoudi Vagourdi ${ }^{1}$, Sk Imran Ali $^{1}$, Silvia Müllner ${ }^{2}$, Peter Lemmens ${ }^{2}$, Reinhard Kremer $^{3}$, Mats Johnsson ${ }^{1}$

${ }^{1}$ Department of Materials and Environmental Chemistry, Stockholm University, Stockholm, Sweden, ${ }^{2}$ Institute of Physics of Condensed Matter, TU Braunschweig, Braunschweig, Germany, ${ }^{3}$ Max Plank Institute for Solid State Research, Stuttgart, Germany E-mail: eleni.mitoudi-vagourdi@mmk.su.se

Single crystals of the new oxo-fluoride CoBi2O2F4 were successfully grown by a hydrothermal technique at $200^{\circ} \mathrm{C}$. The crystal structure was solved from single crystal X-ray diffraction data, collected at different temperatures; $100 \mathrm{~K}, 298 \mathrm{~K}, 243$ $\mathrm{K}$ and $293 \mathrm{~K}$. The compound is layered and crystallizes in the tetragonal non-centrosymmetric space group I-4 where $a=3.843(2) \AA, c=16.34(8) \AA$. Two building blocks make up the crystal structure; [BiO4F4] distorted cubes and distorted [CoF6] octahedra. Interestingly the $\mathrm{F}$ atoms of [CoF6] show four-fold splitting at room temperature. The disorder of the $\mathrm{F}$ atoms is temperature dependent and decreases with decreasing temperature. The [CoF6] octahedra are connected to each other via corner sharing to form [CoF4] $\infty$ layers. In between there are layers of [BiO4F4] polyhedra connected by $\mathrm{Bi}-\mathrm{O}$ bonds to form [BiO2F2] $\infty$ layers where the F-atoms further connect to the [CoF4] $\infty$ layers. Raman scattering data were collected at various temperatures from room temperature to $10 \mathrm{~K}$ in order to investigate the disorder in the structure. Sharper phonon peaks appeared and several modes revealed as the temperature decreases which is an indication of reduction in structural disorder. Magnetic susceptibility and heat capacity measurements showed long range antiferromagnetic ordering below the Néel temperature of $\sim 50 \mathrm{~K}$. The magnetic susceptibility follows the Curie-Weiss law above $75 \mathrm{~K}$ with the Curie Weiss temperature $\theta=-100 \mathrm{~K}$, the Curie constant $\mathrm{C}=3.53 \mathrm{emu} \cdot \mathrm{K} \cdot \mathrm{mol}-1$ and the effective magnetic moment $\mu$ eff $=4.34 \mu \mathrm{B}$.

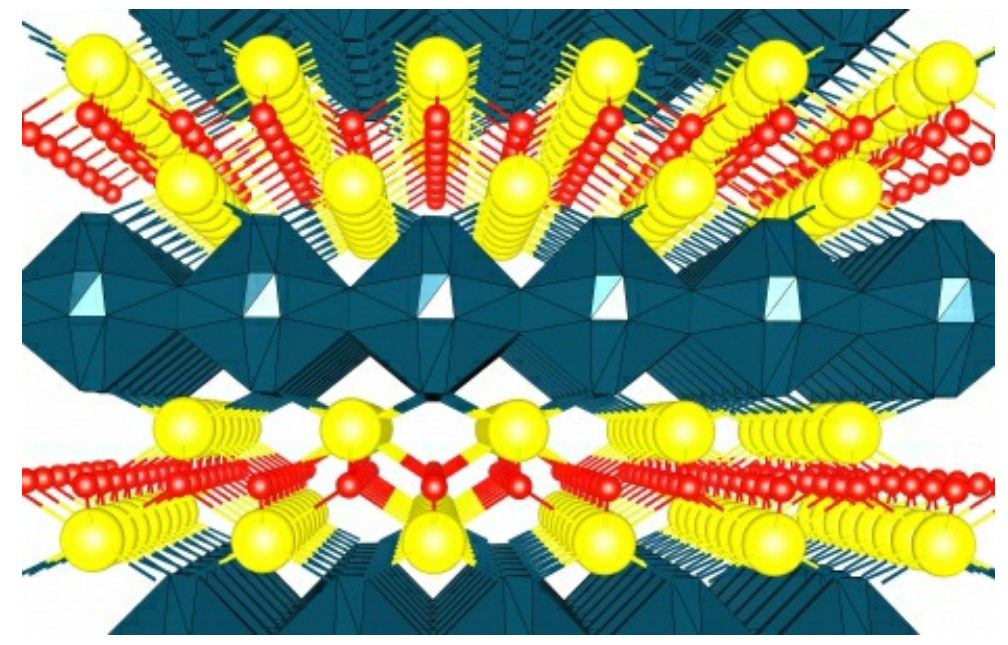

Keywords: Low Dimensional Compounds, Oxofluorides, X-ray Diffraction 